\title{
Infection of human monocyte-derived dendritic cells by ANDES Hantavirus enhances pro- inflammatory state, the secretion of active MMP-9 and indirectly enhances endothelial permeability
}

Delphine Marsac ${ }^{1}$, Stephanie García ${ }^{1}$, Alexandra Fournet ${ }^{2}$, Adam Aguirre $^{3}$, Karla Pino ${ }^{1}$, Marcela Ferres ${ }^{4}$, Alexis M Kalergis ${ }^{5}$, Marcelo Lopez-Lastra ${ }^{1}$ and Francisco Veas ${ }^{2^{*}}$

\begin{abstract}
Background: Andes virus (ANDV), a rodent-borne Hantavirus, is the major etiological agent of Hantavirus cardiopulmonary syndrome (HCPS) in South America, which is mainly characterized by a vascular leakage with high rate of fatal outcomes for infected patients. Currently, neither specific therapy nor vaccines are available against this pathogen. ANDV infects both dendritic and epithelial cells, but in despite that the severity of the disease directly correlates with the viral RNA load, considerable evidence suggests that immune mechanisms rather than direct viral cytopathology are responsible for plasma leakage in HCPS. Here, we assessed the possible effect of soluble factors, induced in viral-activated DCs, on endothelial permeability. Activated immune cells, including DC, secrete gelatinolytic matrix metalloproteases (gMMP-2 and -9) that modulate the vascular permeability for their trafficking.
\end{abstract}

Methods: A clinical ANDES isolate was used to infect DC derived from primary PBMC. Maturation and proinflammatory phenotypes of ANDES-infected DC were assessed by studying the expression of receptors, cytokines and active gMMP-9, as well as some of their functional status. The ANDES-infected DC supernatants were assessed for their capacity to enhance a monolayer endothelial permeability using primary human vascular endothelial cells (HUVEC).

Results: Here, we show that in vitro primary DCs infected by a clinical isolate of ANDV shed virus RNA and proteins, suggesting a competent viral replication in these cells. Moreover, this infection induces an enhanced expression of soluble pro-inflammatory factors, including TNF- $\alpha$ and the active gMMP-9, as well as a decreased expression of anti-inflammatory cytokines, such as IL-10 and TGF- $\beta$. These viral activated cells are less sensitive to apoptosis. Moreover, supernatants from ANDV-infected DCs were able to indirectly enhance the permeability of a monolayer of primary HUVEC.

Conclusions: Primary human DCs, that are primarily targeted by hantaviruses can productively be infected by ANDV and subsequently induce direct effects favoring a proinflammatory phenotype of infected DCs. Finally, based on our observations, we hypothesize that soluble factors secreted in ANDV-infected DC supernatants, importantly contribute to the endothelial permeability enhancement that characterize the HCPS.

\footnotetext{
* Correspondence: francisco.veas@ird.fr

¿UMR-MD3-University Montpellier 1, Comparative Molecular Immuno-

Physiopathology Lab, Faculté de Pharmacie, 34093 Montpellier, France

Full list of author information is available at the end of the article
} 


\section{Background}

Hantaviruses are rodent-born enveloped RNA-viruses belonging to Bunyaviridae family. Two major severe pathologies associated to Hantaviruses have been reported: hemorrhagic fever with renal syndrome (HFRS) in the Eurasia and Hantavirus cardiopulmonary syndrome (HCPS) in the Americas. HCPS is more frequently associated (40\%) to fatal outcomes than HFRS $(<1 \%)$ [1]. Andes Hantavirus (ANDV) is the major etiological agent of the HCPS in South America, syndrome characterized by the presence of high amounts of pulmonary fluids leading to an edema evolving to a cardiogenic shock that synergistically acts with hypovolemia due to capillary leakage resulting in an abrupt cardiopulmonary collapse [2]. Although disease severity directly correlates with the viral RNA load [3], considerable evidence exists suggesting that immune mechanisms rather than direct viral cytopathology are indeed responsible for the massive vascular dysfunction and plasma leakage of HFRS and HCPS $[4,5]$.

The hemorrhagic viruses, including the members of the Bunyaviridae as well as dengue viruses, target endothelial cells and immune cells, mainly monocyte-derived cells such as the professional antigen-presenting cells, Dendritic cells (DCs) [6-8]. DCs activation triggers their maturation and trans-endothelial migration occurring during wound healing or inflammation. These processes require extracellular matrix remodeling and involve changes in endothelial permeability regulated by the production of matrix metalloproteases (gMMPs) or vascular endothelial growth factor (VEGF). However, in excess, these soluble factors can have deleterious effects on endothelial cell integrity. Data from different reports show that endothelial cells infected by dengue virus trigger secretion of soluble factors such as VEGF and the decrease of VEGF-R2 receptor $[9,10]$. We have recently reported in vitro and in vivo showing that soluble factors secreted from DV-infected DCs enhance endothelial permeability and down-regulate expression of endothelial junction proteins, Pecam-1 and VE-cadherin in a gMMP-9-dependent manner [11]. More recently, complementary and convergent studies, to our own previous data on dengue, have reported that Hantavirusinfected endothelial cells enhances the permeability via the reduction of VE-cadherin expression due to its dissociation with VEGF-receptor2 (VEGF-R2) which, in turn, become associated with VEGF $[12,13]$. An accurate understanding of Hantavirus pathogenesis is pivotal to design de novo therapeutic or vaccine approaches that are still lacking against this hemorrhagic viral infection. In this study, we show that ANDV-infected DC are quickly activated and rapidly progress to an intermediate maturation and pro-inflammatory state that contributes to the increase of soluble factors in their supernatant able to trigger the enhancement of endothelial permeability.

\section{Methods \\ Virus and cells}

The primary isolate, ANDV strain CHI-7913 was propagated in the epithelial Vero-E6 cell line (ATCC CRL 1586). Titrated supernatants of these cells were used to infect, at a MOI of 1 for $2 \mathrm{~h}$, human iDCs derived from peripheral blood monocytes (PBMC), as previously described [14]. In these experiments, UV ( $\lambda: 250 \mathrm{~nm}$; 15 min)-irradiated ANDV was used as the negative control. Four days post-DC infection, ANDV N-protein was detected by indirect immunofluorescence (IFA) using a well characterized anti-ANDV $\mathrm{N}$ monoclonal antibody (MAb) [15]. Total RNA was extracted using the High Pure viral nucleic acid kit (Roche Molecular Biochemicals, Mannheim, Germany) following the manufacture's protocol and $1 \mu$ of total RNA was amplified in a one step RTPCR (SuperScript III One-Step RT-PCR with Platinum Taq, Invitrogen) using primers that recognize the nucleocapsid coding region (forward primer: 5' ACA CGA ACA ACA GCT CGT GAC ' 3 and reverse primer: 5' AGG CTC AAG CCC TGT TGG ATC '3). To assess the viral infectivity, from ANDV-positive DCs, their supernatants were used to infect Vero-E6 cells.

\section{Phenotype profiling of ANDES-infected DC}

The influence of ANDV ( $3 \mathrm{~h}$ post-viral) infection of iDCs (as described above) was assessed through the expression of key cell surface proteins CD80, CD83, CD86 and HLA$\mathrm{DR}$, known to be associated with the mature DCs ( $\mathrm{mDCs}$ ) phenotype. Pro-inflammatory profile of ANDV-infected iDCs was assessed through the expression level of some pro and anti-inflammatory cytokines as well as gMMP. As infection of iDCs by other Hantaviruses induces the proinflammatory cytokines expression [7]. Lipopolysaccharide (LPS)-pulsed iDCs were used as a positive control for iDCs maturation, while iDCs incubated with fresh culture medium (mock) were used as a negative control. Supernatants of Mock-DCs, ANDV-infected DCs, and LPS-pulsed DCs were assessed for the production of the pro-inflammatory cytokine, TNF- $\alpha$, (ELISA kit OptEIA ${ }^{\text {TM }}$ Human TNF ELISA Kit II; Pharmingen, Franklin Lakes, NJ).

\section{Functional assessment of ANDV-infected DC}

The effect of ANDV infection on the endocytic capacity of DCs was assessed by measuring the FITC-conjugated Dextran up-take at $37^{\circ} \mathrm{C}$ [16]. In this assay, $18 \mathrm{~h} \mathrm{LPS-}$ pulsed iDCs were used as a control for cell maturation assessment, while iDCs, incubated at $4^{\circ} \mathrm{C}$, were used as a control for the immature stage with lowest capacities 
of endocytosis. The apoptotic level of ANDV-infected DCs was assessed by flow cytometry using the Annexin $\mathrm{V}$ assay [17] using the same previous controls.

\section{Gelatinolytic MMP-9 secretion}

Intra-cellular expression of gMMP-9 in ANDV-infected DCs (3 h-post infection) was characterized by Western blotting, using a mouse anti-gMMP-9 MAb (MAB13416; Chemicon International, Temecula, CA). The gelatinolytic activity of gMMP-9 DC supernatants (SN) was assessed by gelatin zymography [18]. In these experiments fresh medium (SN-Mock) was used as a negative control for maturation, while medium containing LPS $(1 \mu \mathrm{g} / \mathrm{ml})$ (SN-LPS) was used as a positive control.

\section{Endothelial monolayer permeability assessment}

The capacity to modify the vascular permeability of ANDV-infected DC supernatants was measured in vitro, using a monolayer of primary human umbilical vascular endothelial cells (HUVEC) [19] Briefly, HUVEC obtained from pooled donors were plated onto collagen-coated transwell inserts $(0.4 \mu \mathrm{m}$-pore, $6.5 \mathrm{~mm}$-diameter Transwell-COL; Costar) and once in confluence cells, were exposed for $18 \mathrm{~h}$ to mock-SN, virus- free SN from ANDV infected DCs, or to TNF- $\alpha$ (50 nM). Monolayer endothelial permeability was assayed with the standard cell permeability assay according to the Chemicon ECM-640 procedure (Millipore), by adding FITC-conjugated dextran to the upper chamber of the transwell inserts and evaluating the migration of FITC-conjugated Dextran to the lower chamber by using a TECAN fluorometer at $490 / 530 \mathrm{~nm}$. In this transwell system, endothelial monolayer permeability is directly proportional to the flux of $70-\mathrm{kDa}$ dextran passing through the HUVEC monolayer.

\section{Statistical Methods}

Data sets were compared using Kruskal \& Wallis analysis of variance was performed with the GraphPad Prism 4.03 software. Two-sided p-values were considered.

\section{Results and Discussion}

Primary isolate, ANDV strain CHI-7913, was shown to efficiently propagated in the epithelial Vero-E6 cell line (Figure 1A), as previously reported [20], and that their titrated supernatants efficiently targeted and infected human iDCs (Figure 1A). At 4 days post-infection ANDV $\mathrm{N}$-protein was detected by IFA using an anti-ANDV N MAb. Consistent with the cell immunofluorescence data, the presence of ANDV RNA in extensively washed infected DCs was confirmed by an ANDV-specific RT-PCR [21] (Figure 1B). The presence of both, viral antigens and RNA, in DCs does not necessarily imply viral replication, as the observations can be explained by the uptake of exogenous viral particles by cells without productive infection. To check for this option, infected DCs were extensively washed and fresh medium was added. Cell supernatants were recovered, total RNA isolated as described above and subjected to an ANDV-specific RT-PCR. The presence of viral RNA in DC supernatants was confirmed (Figure 1C), suggesting shedding of de novo generated viral particles. To assess the viral infection capacity, supernatants from ANDV-positive DCs were used to infect Vero-E6 cells. Five days post-infection, the presence of ANDV in Vero-E6 cells was confirmed by both IFA and RT-PCR as described above (data not shown). Together these observations strongly suggest that, consistent with other members of the Hantavirus genus of the Bunyaviridae, ANDV is able to infect and replicate in human DCs. Consequently, we explored the possible effects of this viral infection on both DC phenotypes and functionalities.

The impact of DC infection by ADNV on cell viability, maturation and endocytosis was assessed. The apoptotic level, evaluated by flow cytometry using the Annexin V assay [17], showed that infection of iDCs with ANDV does not affect cell viability (Figure 2A), observations in line with what has been reported for other hantaviruses $[6,22]$. In addition, ANDV infection did not induce any detectable cytopathic effect (data not shown). DCs play a pivotal role as antigen-presenting cells in the antiviral immune response. It is known that infection of iDCs by diverse viruses stimulates cell homing to inflammatory sites as well as their maturation into antigen-presenting cells (APC), a process essential for the initiation and modulation of $\mathrm{T}$ cell-mediated immune responses. Hence, we next evaluated whether ANDV infection of iDCs had a direct effect on the expression of key cell surface proteins CD80, CD83, CD86 and HLA-DR, known to be associated with a mature DCs (mDCs) phenotype. Immature DCs were infected with ANDV as described above. ANDV infection of iDCs induced a significant increase of HLA$\mathrm{DR}$, a marker protein that plays a pivotal role in guiding the development and activation of $\mathrm{CD} 4^{+} \mathrm{T}$ helper cells. Markers CD83 and CD86 also increased, albeit to a much lower extent than in LPS-pulsed iDCs conditions. As expected, expression of all surface markers increased in LPS-pulsed iDCs as compared to mock-DCs (Figure 2B). These data suggest that ANDV infection of human DCs induced cell maturation, and that ANDV infection alters the expression of HLA-DR on DCs. Constitutive expression of HLA-DR molecules is associated to professional antigens presenting cells (APCs) such as DCs, this basal expression can be enhanced in an environment enriched with proinflammatory cytokines $[7,23]$.

Immature DCs efficiently capture and process antigen, a characteristic cell function that decreases with maturation [7,8,24-27]. Therefore, we next sought to establish whether the partial DC maturation phenotype (Figure 2B) was correlated with its decreased ability to capture antigens. To 

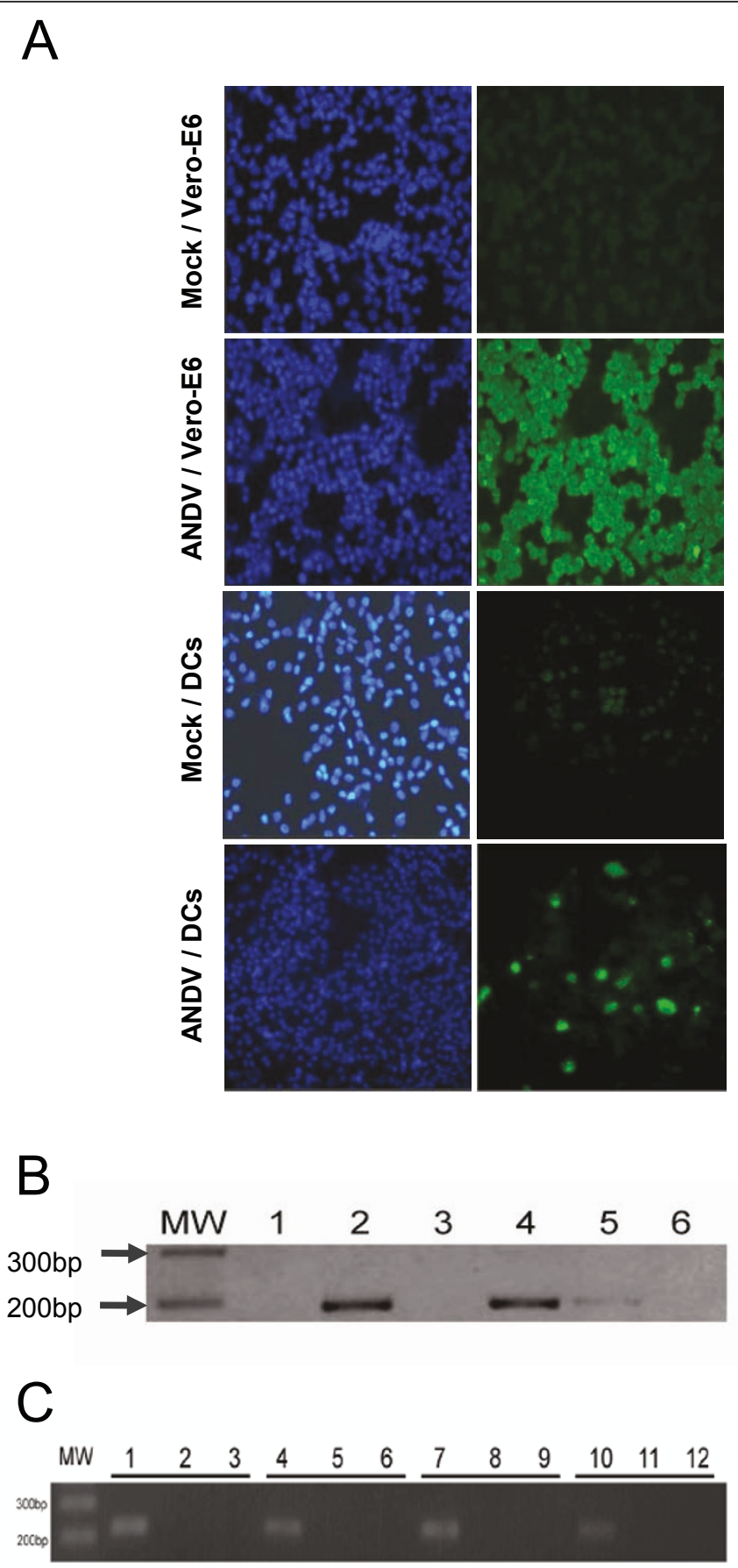

Figure 1 Detection of ANDV infection of primary human dendritic cells. (A) Vero E6 epithelial cells and human immature DCs infected with ADNV (strain CHI-7913). ANDV-nucleocapsid (N) protein detected by IFA by incubating ANDV-infected with a mouse anti-ANDV N MAb revealed by an FITC-conjugated anti-mouse IgG Ab (Green, right column) while the corresponding cell nuclei were stained with 4',6-diamidino-2phenylindole (DAPI, blue, left column). Negative control (mock) cells were incubated with supernatant from uninfected Vero-E6 cells. (B) Total RNA was extracted from uninfected LPS-Pulsed DCs (lane 1), ANDV-infected LPS-pulsed DCs (lane 2), uninfected iDCs (lane 3), ANDV-infected iDCs (lane 4), ANDV-infected Vero-E6 cells (lane 5), and used as template in a RT-PCR reaction designed to specifically amplify the viral S RNA. This assay also included a negative RT-PCR control (lane 6). MW is a molecular weight marker (1 Kb, Fermentas, Burlington, Canada). (C) DCs generated from primary monocytes, recovered from four healthy donors, were incubated with ANDV (lanes 1, 4, 7, 10), UV-irradiated ANDV (lanes $2,5,8,11)$ or pulsed with LPS (lanes $3,6,9,12)$. Total RNA was extracted from cell supernatants and used as a template in a RT-PCR reaction designed to specifically amplify the viral S RNA. MW is a molecular weight marker (100 pb, Fermentas). 
A

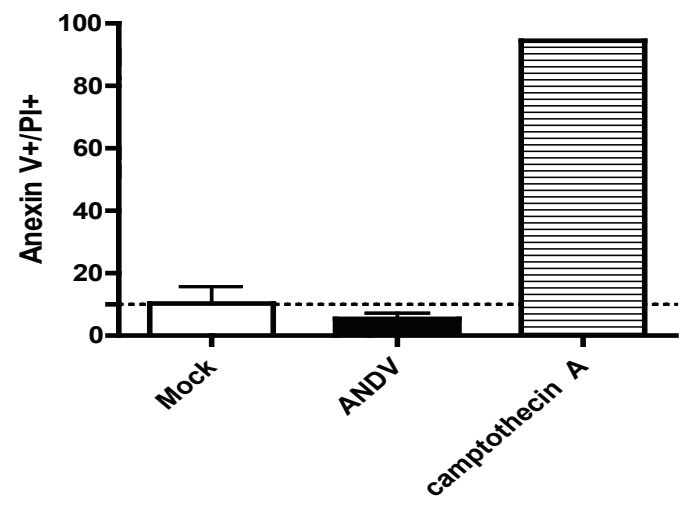

B
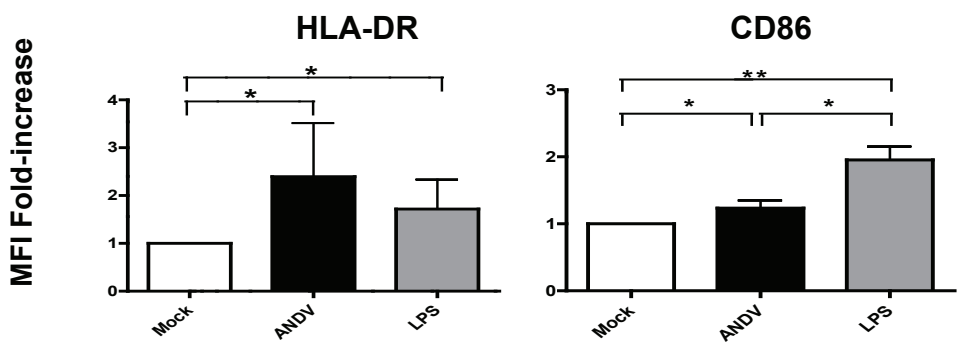

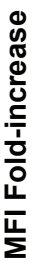

CD80
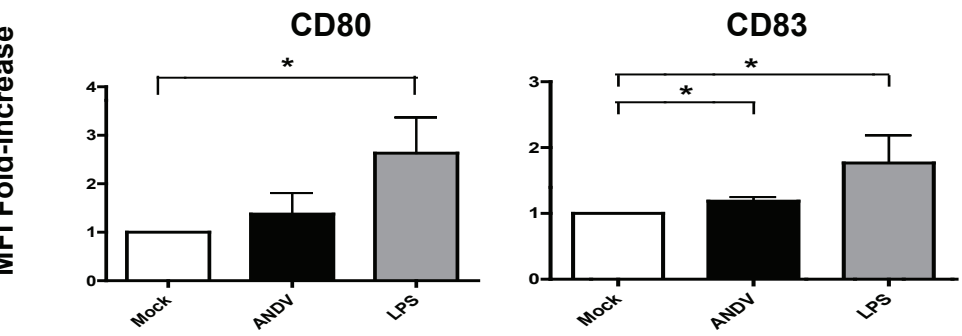

C

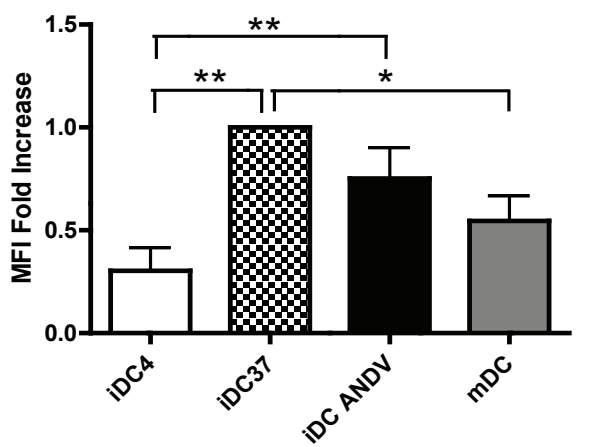

Figure 2 Impact of ANDES virus infection on iDC phenotype and functions. (A) The impact of ANDV infection on DCs viability was detected by using the Annexin V-propidium iodide (PI) method. In these assays, DCs treated with camptothecin A (4 $\mu \mathrm{M}$ for $18 \mathrm{~h}$ ) were used as a positive apoptosis control. (B) DCs surface markers; CD80, CD86, CD83 and HLA-DR were analyzed in ANDV-infected iDCs by flow cytometry four days post-ANDV infection, while LPS-pulsed DCs and uninfected iDCs (mock) were used as controls. Bar graphs represent the fold-increase expression of these surface markers as compared their expression in mock control. Data are means of three independent experiments: ${ }^{*}, p<0.05$; **, $p<0.01$. (C) The endocytic capacity of ANDES infected iDCs, LPS-matured DCs (mDC), uninfected iDCs incubated at $37^{\circ} \mathrm{C}$ (iDC 37 ), and uninfected iDCs incubated at $4^{\circ} \mathrm{C}$ (iDC 4) was assessed using a FITC-conjugated Dextran ( $30 \mu \mathrm{g}$ ). Endocytosis was analyzed by flow cytometry after $2 \mathrm{~h}$ of incubation. Mean fluorescence intensity values within the gate for the different endocytically active stages were plotted. Bar graphs show the fold-increases of the mean fluorescence intensities (MFI), relative to the mock control (iDCs 37). For each experiment, 10000 gated cells were evaluated. Data are means of five independent experiments. ${ }^{*}, p<0.05 ;{ }^{* *}, p<0.01$. 
this purpose, we studied the effect of ANDV infection on the endocytic capacity of DCs by measuring the up-take of FITC-conjugated Dextran at $37^{\circ} \mathrm{C}$ [16]. Results indicate that ANDV-infected DC exhibited a reduced endocytic capacity as compared to uninfected iDCs (Figure 2C). However, in our conditions, infected DCs exhibited a higher endocytic activity than the used dose of LPS to pulse iDCs (positive control). Together, these observations suggest that ANDV infection does indeed reduce antigen capture by DCs, supporting a role of ANDV infection in cell maturation.

The presence of high levels of pro-inflammatory cytokines both in plasma and lungs have been reported as pathological markers associated with HCPS in humans. These excesses of pro-inflammatory cytokines are secreted in patients by both hantavirus-activated macrophages and specific T cells [28]. Particularly, the levels of the proinflammatory cytokine TNF- $\alpha$ in HCPS patient sera are the dramatically elevated $[29,30]$. Infection of iDCs by other Hantaviruses induces the production of pro-inflammatory cytokines as well [7]. Therefore, we sought to evaluate whether ANDV infection induces a similar DC phenotype. Supernatants of Mock-DCs, ANDV-infected DCs (3 h post-viral infection), and LPS-pulsed DCs were assessed for the production of the pro-inflammatory cytokine, TNF- $\alpha$. In agreement with what has been described for other Hantaviruses, supernatants harvested from ANDV-infected DCs display a significant increase in TNF- $\alpha$ as compared with supernatants from uninfected iDCs (Figure 3A). In a similar experimental setting, we also evaluated the expression of IL-10 and TGF- $\beta$, two anti-inflammatory cytokines [31]. Interestingly, in supernatants harvested from ANDV-infected DCs, IL-10 levels were weakly altered as compared with uninfected DCs, while TGF- $\beta$ levels decrease was more pronounced (Figure $3 \mathrm{~B}$ and $3 \mathrm{C}$ ). Thus, our observations suggest that ANDV-infected DCs exhibit a pro-inflammatory cytokine profile.

Upon antigen capture, DCs undergo a process of maturation and a lymph nodes homing, where they activate the adaptive immune system. The process of DC trafficking is complex. This phenomenon requires the enhancement of the vascular permeability, which could involve the expression of several components, such as cell to cell junction proteins including VE-cadherin, PECAM-1, occludin, claudins, as well as soluble factors, including, IL-1 $\beta$, TNF- $\alpha$, VEGF, and Kinins [32-34]. The gelatinolytic matrix metalloproteases (gMMP)-2 and 9 are reported as one of the major actors of this crucial phenomenon during fetal development and wound healing [35]. Interestingly, factors such as the inflammatory cytokines IL-8, TNF- $\alpha$ and IL- $1 \beta$ as well as VEGF [36] are in the upstream of the gMMP expression pathway in human monocytes [37]. Additionally, even if iDC produces gMMP, DC increase the MMP-9 production along

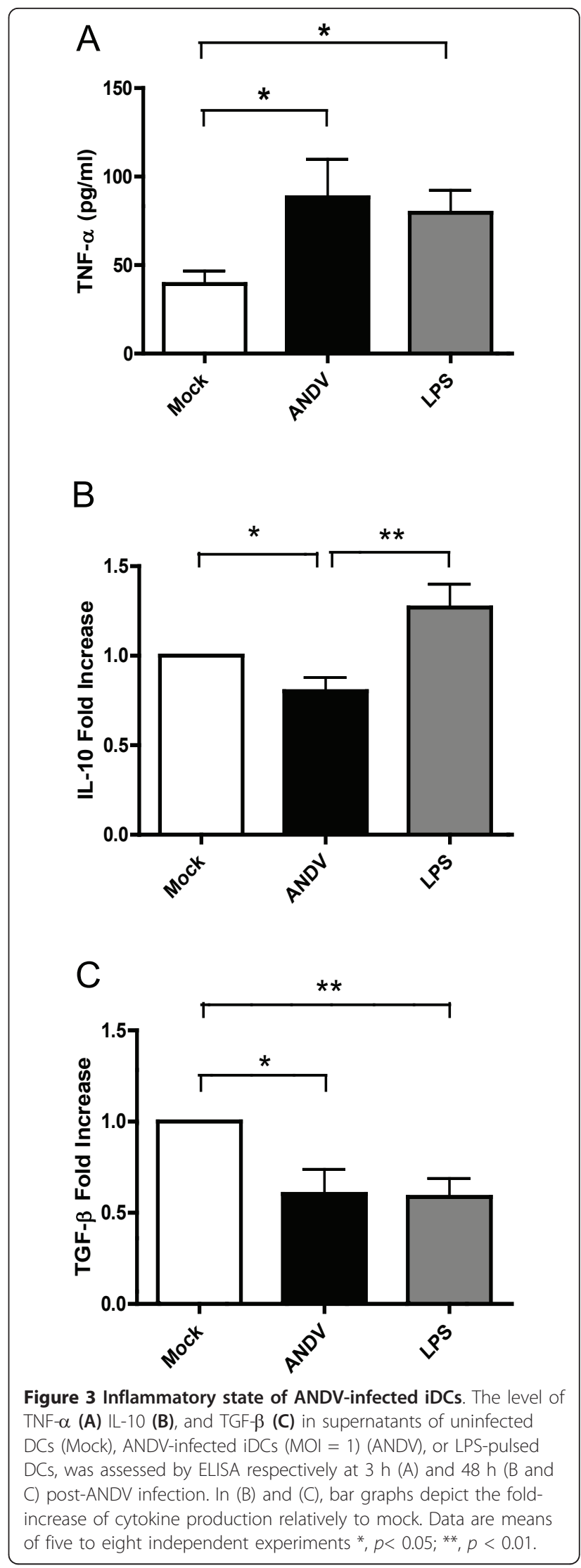


DC maturation progression [38]. Interestingly, certain viruses can play opposite roles in DC maturation, for example the human Cytomegalovirus (hCMV) inhibits cell maturation to escape the immune system (4), whereas some others, such as HIV-1 [18], Dengue virus [11], and West Nile virus [39], enhance DC maturation, the secretion of active gMMP-9, as well as plasma vascular leakage. Thus, to extend our previous observation on the fact that DCs have a proinflammatory profile that can be compatible with the secretion of gMMP-9, we analyzed the expression and activity of gMMP-9 in cells and its presence in DC supernatants (SN). Selection seemed adequate as the levels of IL-10, which has been reported to inhibit MMP-9 induction, is weakly altered by ADNV infection (Figure 3B) and DC maturation is known to increase the level of active MMP-9 secretion. Intra-cellular expression of gMMP-9 in ANDV-infected DCs (3 hpost infection) was characterized by Western blotting (Figure 4A), using a mouse an anti-gMMP-9 MAb. Data show that gMMP-9 expression is indeed elevated in ANDV-infected DCs as compared with mock iDCs (Figure 4A). Gelatinolytic MMP-9 is secreted as a proenzyme, which remains inactive unless it is activated by the removal of the propeptide domain by proteolytic enzymes. The gelatinolytic activity of gMMP-9 was therefore assessed in cell supernatants $(\mathrm{SN})$ by gelatin zymography [18]. Strikingly, ANDV-infected DC supernatants exhibited an elevated gMMP-9 activity as compared with SN-mock (Figure 4B). Furthermore, gMMP-9 activity was similar to that obtained by the LPS-induction of DC maturation (SN-LPS; Figure 4B).

ANDV belongs to the group of hemorrhagic fever viruses known for inducing different forms of vascular leakage in different organs. For some of these hemorrhagic fever or encephalitic viruses the mechanism associated to their capacity to enhance endothelial permeability in vitro and in vivo has been reported $[11,18,39]$. Upon viral infection, the vascular permeability phenomenon is principally due to viral-dependent dysfunctions of endothelial cells junctions that can be directly induced by the action of factors from viral activated endothelial cells, such as VEGF or indirectly induced by factors from immune viral-activated cells, via the MAP-kinase pathway $[11,18]$. For these reasons, we wonder if ANDV infection induced the production of soluble factors that could indirectly enhance endothelial permeability. To evaluate this possibility, the capacity of ANDV infected DCs-supernatants to modify the permeability was measured through the amounts of FITC-conjugated dextran that passed thru a monolayer of primary human umbilical vascular endothelial cells (HUVEC) seeded onto transwell plates that considered as vascular model [19]. Following the exposure of confluent HUVEC to SN from ANDV infected DCs, or to controls, the amounts of FITC-conjugated dextran detected in the
A

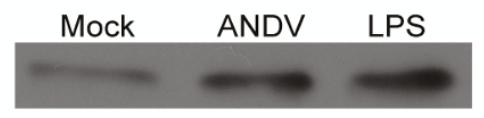

B
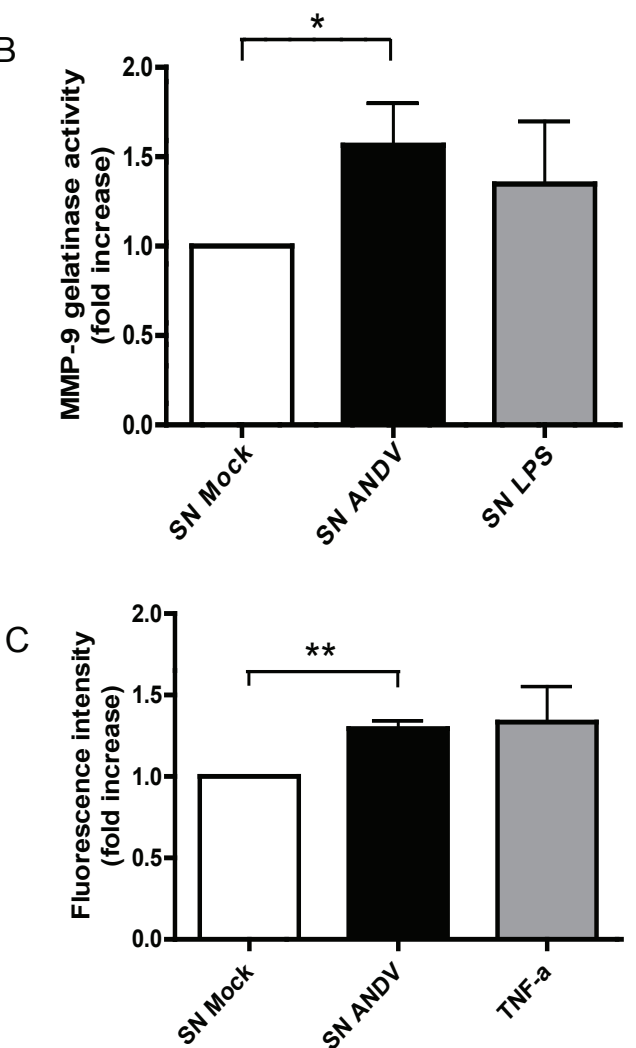

Figure 4 Secretion of active gMMP-9 and endothelial permeability enhancement are triggered by ANDV infection of iDCs. (A) The gMMP-9 expression in ANDV-infected iDCs was assessed by Western blotting. Uninfected iDCs (Mock), ANDVinfected iDCs (ANDV) and LPS-treated iDCs (LPS) were collected $3 \mathrm{~h}$ post-infection. Forty micrograms of total proteins from cell lysates were separated in an 8\% SDS-PAGE gel. Following an electrical protein transfer onto a nitrocellulose membrane, an anti-gMMP-9 antibody was used to probe the presence of gMMP-9 that was revealed using an HRP-conjugated anti-mouse IgG Ab. (B) Supernatants from ANDV-infected DCs or Mock cells collected $3 \mathrm{~h}$ post-infection. Gelatinase activity was assayed by zymography. Gelatinolytic activity was quantified by gel densitometry using the Image J Software. Data are presented as the fold-increase of gMMP9 activity in supernatants. Statistical significance $(* *, p<0.01)$ was determined from five independent experiments. (C) Enhancement of the endothelial cells permeability induced by supernatant from ANDV-infected iDCs. HUVEC confluent monolayers plated onto collagen-coated transwell inserts were incubated with either ANDV supernatant, mock control (uninfected DCs supernatant) or with TNF- $\alpha(50 \mathrm{ng} / \mathrm{ml})$ as positive control. Following $18 \mathrm{~h}$ at $37^{\circ} \mathrm{C}$ in $\mathrm{CO} 2$ $5 \%$ after addition, within the top chamber, of $500 \mu \mathrm{g} / \mathrm{ml} \mathrm{FITC-}$ conjugated Dextran, paracellular permeability was measured by reading in the bottom chamber containing the infiltrated FITCdextran at an excitation wavelength of $485 \mathrm{~nm}$ and an emission of $530 \mathrm{~nm}$. Data represent means of five independent experiments. **, $p<0.01$. 
lower chamber of the transwell system (Figure 4C) suggest that cell supernatant from ANDV-infected DCs contains soluble and active factors favoring the increase of the HUVEC monolayer permeability.

Altogether, the in vitro data presented herein show that ANDV infects and replicates in DCs, inducing a partial maturation and pro-inflammatory phenotype, while increasing the expression and secretion of active gMMP-9 as well as that supernatants from ANDVinfected DCs were able to enhance the permeability of a monolayer of primary human vascular endothelial cells.

\section{Conclusion}

The herein presented data and data recently published in the literature are not only convergent but also complementary, they allow to speculate that both indirect effects of ANDV on DCs and direct effects of pathogenic hantaviruses, such as ANDV, on endothelial cells generate conjugated inflammatory consequences that result in the increased endothelial permeability associated to this virus infection. A better understanding of major mechanisms involved in vascular pathogenesis observed in HCPS is pivotal to progress in the design of therapies that today remain unavailable for this serious life-threatening hemorrhagic viral disease.

\section{Acknowledgements}

We thank Dr. M. Rau (Oxford, UK) for critical reading and editing of the manuscript. We are grateful to Dr. Nicole Tischler, Fundación Ciencias para la Vida, Santiago-Chile, for kindly providing the anti-N MAb used in this study. We thank colleagues from the Pontificia Universidad Católica de Chile: Prof. Roberto Ebenspenger and Lorena Rubio for kindly providing HUVECs; Connie Martinez for titrated viruses. The present study was supported by CONICYT through grant FONDECYT-POSTDOCTORADO-2008-3085029 to DM, FONDECYT No 1100756, and PHS grant 2U01AI045452-11 to MLL, Proyecto Instituto Milenio P-07-088-F to AK and MLL, Chile. We also thank IRD and University Montpellier 1, France, for supporting a part of this study.

\section{Author details}

${ }^{1}$ Pontificia Universidad Católica de Chile, Millennium Institute on Immunology and Immunotherapy, Laboratorio de Virología Molecular, Facultad de Medicina, Santiago, Chile. ' $U$ MR-MD3-University Montpellier 1, Comparative Molecular Immuno-Physiopathology Lab, Faculté de Pharmacie, 34093 Montpellier, France. ${ }^{3}$ Universidad de Chile, Laboratorio de Comunicaciones Celulares. Centro de Estudios Moleculares de la Célula, Facultad de Medicina, Santiago, Chile. ${ }^{4}$ Pontificia Universidad Católica de Chile, Laboratorio de Infectologia, Centro de Investigaciones Medicas, Facultad de Medicina, Santiago, Chile. ${ }^{5}$ Pontificia Universidad Católica de Chile, Millennium Institute on Immunology and Immunotherapy. Departamento de Genética Molecular y Microbiología, Facultad de Ciencias Biológicas, Santiago, Chile.

\section{Authors' contributions}

DM carried out the BSL3 experiments, hantavirus cultivation, and most of the experiments described in this paper, manuscript draft; SG participated to all of those experiments; AF zymograms and experimental permeability studies set-up. AA performed the statistical analysis. KP RT-PCR; MF BSL-3 facilities; AK DCs PBMC-derived and draft revision; MLL data analysis and manuscript revision; FV designed the project, data interpretation, manuscript revisions, finalized and approved the manuscript. All authors read and approved the final manuscript.

\section{Authors' informations}

DM was a FONDECYT post-doctorate fellowship; SG is PhD student and KP is a technician at the MLL lab; AF is from the technical staff of the FV lab.

\section{Competing interests}

The authors declare that they have no competing interests.

Received: 1 April 2011 Accepted: 13 May 2011 Published: 13 May 2011

\section{References}

1. Hjertqvist M, Klein SL, Ahlm C, Klingstrom J: Mortality rate patterns for hemorrhagic fever with renal syndrome caused by Puumala virus. Emerg Infect Dis 16:1584-1586.

2. Nolte KB, Feddersen RM, Foucar K, Zaki SR, Koster FT, Madar D, Merlin TL, McFeeley PJ, Umland ET, Zumwalt RE: Hantavirus pulmonary syndrome in the United States: a pathological description of a disease caused by a new agent. Hum Pathol 1995, 26:110-120.

3. Xiao R, Yang S, Koster F, Ye C, Stidley C, Hjelle B: Sin Nombre viral RNA load in patients with hantavirus cardiopulmonary syndrome. J Infect Dis 2006, 194:1403-1409.

4. Maes P, Clement J, Gavrilovskaya I, Van Ranst M: Hantaviruses: immunology, treatment, and prevention. Viral Immunol 2004, 17:481-497.

5. Mertz GJ, Hjelle BL, Bryan RT: Hantavirus infection. Adv Intern Med 1997, 42:369-421.

6. Pensiero MN, Sharefkin JB, Dieffenbach CW, Hay J: Hantaan virus infection of human endothelial cells. J Virol 1992, 66:5929-5936.

7. Raftery MJ, Kraus AA, Ulrich R, Kruger DH, Schonrich G: Hantavirus infection of dendritic cells. J Virol 2002, 76:10724-10733.

8. Zaki SR, Greer PW, Coffield LM, Goldsmith CS, Nolte KB, Foucar K, Feddersen RM, Zumwalt RE, Miller GL, Khan AS, et al: Hantavirus pulmonary syndrome. Pathogenesis of an emerging infectious disease. Am J Pathol 1995, 146:552-579.

9. Sathupan P, Khongphattanayothin A, Srisai J, Srikaew K, Poovorawan Y: The role of vascular endothelial growth factor leading to vascular leakage in children with dengue virus infection. Ann Trop Paediatr 2007, 27:179-184.

10. Srikiatkhachorn A, Ajariyakhajorn C, Endy TP, Kalayanarooj S, Libraty DH, Green S, Ennis FA, Rothman AL: Virus-induced decline in soluble vascular endothelial growth receptor 2 is associated with plasma leakage in dengue hemorrhagic Fever. J Virol 2007, 81:1592-1600.

11. Luplertlop N, Misse D, Bray D, Deleuze V, Gonzalez JP, Leardkamolkarn V, Yssel H, Veas F: Dengue-virus-infected dendritic cells trigger vascular leakage through metalloproteinase overproduction. EMBO Rep 2006, 7:1176-1181.

12. Gorbunova E, Gavrilovskaya IN, Mackow ER: Pathogenic hantaviruses Andes virus and Hantaan virus induce adherens junction disassembly by directing vascular endothelial cadherin internalization in human endothelial cells. J Virol 84:7405-7411.

13. Shrivastava-Ranjan P, Rollin PE, Spiropoulou CF: Andes virus disrupts the endothelial cell barrier by induction of vascular endothelial growth factor and downregulation of VE-cadherin. J Virol 84:11227-11234.

14. Sallusto F, Lanzavecchia A: Efficient presentation of soluble antigen by cultured human dendritic cells is maintained by granulocyte/ macrophage colony-stimulating factor plus interleukin 4 and downregulated by tumor necrosis factor alpha. J Exp Med 1994 179:1109-1118.

15. Tischler ND, Rosemblatt M, Valenzuela PD: Characterization of crossreactive and serotype-specific epitopes on the nucleocapsid proteins of hantaviruses. Virus Res 2008, 135:1-9.

16. Lutz MB, Rovere $P$, Kleijmeer MJ, Rescigno M, Assmann CU, Oorschot VM, Geuze HJ, Trucy J, Demandolx D, Davoust J, Ricciardi-Castagnoli P. Intracellular routes and selective retention of antigens in mildly acidic cathepsin D/lysosome-associated membrane protein-1/MHC class IIpositive vesicles in immature dendritic cells. J Immunol 1997, 159:3707-3716.

17. Nicoletti I, Migliorati G, Pagliacci MC, Grignani F, Riccardi C: A rapid and simple method for measuring thymocyte apoptosis by propidium iodide staining and flow cytometry. J Immunol Methods 1991, 139:271-279.

18. Misse D, Esteve PO, Renneboog B, Vidal M, Cerutti M, St Pierre Y, Yssel H, Parmentier M, Veas F: HIV-1 glycoprotein 120 induces the MMP-9 cytopathogenic factor production that is abolished by inhibition of the 
p38 mitogen-activated protein kinase signaling pathway. Blood 2001, 98:541-547.

19. Jaffe EA, Nachman RL, Becker CG, Minick CR: Culture of human endothelial cells derived from umbilical veins. Identification by morphologic and immunologic criteria. J Clin Invest 1973, 52:2745-2756.

20. Galeno H, Mora J, Villagra E, Fernandez J, Hernandez J, Mertz GJ, Ramirez E: First human isolate of Hantavirus (Andes virus) in the Americas. Emerg Infect Dis 2002, 8:657-661.

21. Godoy $P$, Marsac D, Stefas E, Ferrer P, Tischler ND, Pino K, Ramdohr P, Vial P, Valenzuela PD, Ferres $M$, et al: Andes virus antigens are shed in urine of patients with acute hantavirus cardiopulmonary syndrome. J Virol 2009, 83:5046-5055

22. Yanagihara R, Silverman DJ: Experimental infection of human vascular endothelial cells by pathogenic and nonpathogenic hantaviruses. Arch Virol 1990, 111:281-286.

23. Holling TM, Schooten E, van Den Elsen PJ: Function and regulation of MHC class II molecules in T-lymphocytes: of mice and men. Hum Immunol 2004, 65:282-290.

24. Morelli AE, Thomson AW: Dendritic cells: regulators of alloimmunity and opportunities for tolerance induction. Immunol $\operatorname{Rev} 2003$, 196:125-146.

25. Gavrilovskaya IN, Peresleni T, Geimonen E, Mackow ER: Pathogenic hantaviruses selectively inhibit beta3 integrin directed endothelial cell migration. Arch Virol 2002, 147:1913-1931.

26. Hayasaka D, Maeda K, Ennis FA, Terajima M: Increased permeability of human endothelial cell line EA.hy926 induced by hantavirus-specific cytotoxic T lymphocytes. Virus Res 2007, 123:120-127.

27. Temonen M, Vapalahti $\mathrm{O}$, Holthofer $\mathrm{H}$, Brummer-Korvenkontio M, Vaheri $\mathrm{A}$, Lankinen H: Susceptibility of human cells to Puumala virus infection. J Gen Virol 1993, 74(Pt 3):515-518.

28. Mori M, Rothman AL, Kurane I, Montoya JM, Nolte KB, Norman JE, Waite DC, Koster FT, Ennis FA: High levels of cytokine-producing cells in the lung tissues of patients with fatal hantavirus pulmonary syndrome. $J$ Infect Dis 1999, 179:295-302.

29. Borges AA, Campos GM, Moreli ML, Moro Souza RL, Saggioro FP, Figueiredo GG, Livonesi MC, Moraes Figueiredo LT: Role of mixed Th1 and Th2 serum cytokines on pathogenesis and prognosis of hantavirus pulmonary syndrome. Microbes Infect 2008, 10:1150-1157.

30. Linderholm M, Ahlm C, Settergren B, Waage A, Tarnvik A: Elevated plasma levels of tumor necrosis factor (TNF)-alpha, soluble TNF receptors, interleukin (IL)-6, and IL-10 in patients with hemorrhagic fever with renal syndrome. J Infect Dis 1996, 173:38-43.

31. Carvajal CA, Herrada AA, Castillo CR, Contreras FJ, Stehr CB, Mosso LM Kalergis AM, Fardella CE: Primary aldosteronism can alter peripheral levels of TGF-betaand TNF-alpha. J Endocrinol Invest 2009, 32:759-65.

32. Donmez G, Sullu Y, Baris S, Yildiz L, Aydin O, Karagoz F, Kandemir B: Vascular endothelial growth factor (VEGF), matrix metalloproteinase-9 (MMP-9), and thrombospondin-1 (TSP-1) expression in urothelial carcinomas. Pathol Res Pract 2009, 205:854-857.

33. Imamura T, Tanase S, Hayashi I, Potempa J, Kozik A, Travis J: Release of a new vascular permeability enhancing peptide from kininogens by human neutrophil elastase. Biochem Biophys Res Commun 2002, 294:423-428.

34. Lehmann W, Edgar CM, Wang K, Cho TJ, Barnes GL, Kakar S, Graves DT, Rueger JM, Gerstenfeld LC, Einhorn TA: Tumor necrosis factor alpha (TNFalpha) coordinately regulates the expression of specific matrix metalloproteinases (MMPS) and angiogenic factors during fracture healing. Bone 2005, 36:300-310.

35. Bartholome EJ, Van Aelst I, Koyen E, Kiss R, Willems F, Goldman M, Opdenakker G: Human monocyte-derived dendritic cells produce bioactive gelatinase B: inhibition by IFN-beta. J Interferon Cytokine Res 2001, 21:495-501.

36. Valable S, Montaner J, Bellail A, Berezowski V, Brillault J, Cecchelli R, Divoux D, Mackenzie ET, Bernaudin M, Roussel S, Petit E: VEGF-induced BBB permeability is associated with an MMP-9 activity increase in cerebral ischemia: both effects decreased by Ang-1. J Cereb Blood Flow Metab 2005, 25:1491-1504

37. Saren P, Welgus HG, Kovanen PT: TNF-alpha and IL-1 beta selectively induce expression of $92-k D a$ gelatinase by human macrophages. $J$ Immunol 1996, 157:4159-4165.
38. Osman M, Tortorella M, Londei M, Quaratino S: Expression of matrix metalloproteinases and tissue inhibitors of metalloproteinases define the migratory characteristics of human monocyte-derived dendritic cells. Immunology 2002, 105:73-82.

39. Verma S, Lo Y, Chapagain M, Lum S, Kumar M, Gurjav U, Luo H, Nakatsuka A, Nerurkar VR: West Nile virus infection modulates human brain microvascular endothelial cells tight junction proteins and cell adhesion molecules: Transmigration across the in vitro blood-brain barrier. Virology 2009, 385:425-433.

doi:10.1186/1743-422X-8-223

Cite this article as: Marsac et al:: Infection of human monocyte-derived dendritic cells by ANDES Hantavirus enhances pro-inflammatory state, the secretion of active MMP-9 and indirectly enhances endothelial permeability. Virology Journal 2011 8:223.

\section{Submit your next manuscript to BioMed Central and take full advantage of:}

- Convenient online submission

- Thorough peer review

- No space constraints or color figure charges

- Immediate publication on acceptance

- Inclusion in PubMed, CAS, Scopus and Google Scholar

- Research which is freely available for redistribution

Submit your manuscript at www.biomedcentral.com/submit
Biomed Central 